\title{
Möglichkeiten pharmakologischer Interventionen: Impfstoffe und Pharmakotherapie in der Sucht- behandlung der Zukunft
}

\begin{abstract}
Zusammenfassung: In den letzten Jahren ist es uns gelungen neue Tiermodelle zu entwickeln, die viele Aspekte von Alkoholismus und Opiatabhängigkeit darstellen (nach DSMIV). Mit Hilfe des Alkoholtrinkmodells konnte die Wirksamkeit von Acamprosat (Campral ${ }^{\circledR}$ ) und Naltrexon (Revia ${ }^{\mathrm{TM}}$ ) nachgewiesen werden. Mit weiteren verhaltenspharmakologischen Untersuchungen und elektrophysiologischen / molekularbiologischen Methoden konnten wir den Wirkmechanismus dieser Substanzen weitgehend aufklären: Naltrexon blockiert Opiatrezeptoren auf Neuronen des mesolimbischen Belohnungssystems und hemmt dabei die akuten wie auch konditionierten Verstärkereigenschaften von Alkohol und Opiaten („positives Craving“), während Acamprosat vorwiegend mit dem glutamatergen N-Methyl-D-Aspartat-(NMDA)System interagiert und zu deutlichen Veränderungen genomisch vermittelter Prozesse führt. Acamprosat unterdrückt dabei sowohl akute als auch konditionierte Entzugssymptome („negatives Craving“). Sollte es nun weiterhin möglich werden, bei alkoholkranken Patienten zu unterscheiden zwischen denen, die positives Craving, und solchen, die negatives Craving (o. beides) nach einer „Cue exposure“ verspüren, so könnte eine zielgerichtete Pharmakotherapie eingesetzt werden (individuell adaptierte Pharmakotherapie). Alternativ hierzu werden verschiedene Aspekte der Drogenimmunisierung diskutiert.
\end{abstract}

Schlüsselwörter: Sucht - Rückfall - Craving - Tiermodelle Alkohol - Opiate - Acamprosat - Naltrexon Drogenimmunisierung

Possibilities of Pharmacological Interventions: Vaccines and Pharmacotherapy in the Addiction Treatment of the Future: New animal models of alcoholism and opiate addiction have been developed. Rats derived from our longterm alcohol drinking model show certain symptoms also seen in human alcoholics. Recently, naltrexone and acamprosate have been approved in several countries as relapse prevention in weaned alcoholics. We examined the efficacy of these compounds in our drinking model and tried to elucidate their putative mode of action. The opioid receptor antagonist naltrexone reduced the alcohol-deprivation effect in long-term ethanol experienced rats. It is suggested that naltrexone reduces ethanol reinforcement and conditioned cue reactivity most likely via a blockade of opioid receptors („positive craving“). The repeated administration of acamprosate also reduces the alcohol-deprivation effect. Acamprosate in-

Suchttherapie Sonderheft 2001; 2: S32-S33

(c) Georg Thieme Verlag Stuttgart · New York ISSN 1439-9903
Rainer Spanagel

Zentralinstitut für Seelische Gesundheit, Abteilung Psychopharmakologie terferes with several symptoms of withdrawal most likely via a normalisation of neuronal hyperactivity. It is suggested that acamprosate reduces neuronal hyperactivity during protracted withdrawal and conditioned withdrawal responses via a selective influence upon NMDA-receptor composition ("negative craving“). In the near future we are aiming for an indivual adapted pharmacotherapy where patients who show positive and negative craving, respectively, will be matched to the appropriate treatment. Alternatively to these pharmacological intervention strategies the possibility of drug immunization will be discussed.

Key words: Addiction - Relapse - Craving - Animal Models - Alcohol - Opioids - Acamprosate - Naltexone - Drug Immunization

Der Missbrauch von Alkohol sowie von Opiaten führt zu körperlicher und psychischer Abhängigkeit. Durch die Anpassung des zentralen Nervensystems an die dauernde Drogenzufuhr treten beim Absetzen der Droge Entzugserscheinungen auf. Das Bedürfnis des Süchtigen, seine Entzugserscheinungen zu lindern bzw. ihrem Auftreten durch fortgesetzten Drogenkonsum zuvorzukommen, wird von den meisten Suchtexperten als eine wichtige Motivation zum weiteren Drogenmissbrauch angesehen. Ein entscheidender auslösender Faktor für ein Rückfallverhalten aber ist das Phänomen eines starken, teilweise als übermächtig empfundenen Verlangens („Craving“) nach der Droge. Selbst nach jahrelanger Drogenabstinenz kann dieses plötzlich wieder auftreten und einen Rückfall auslösen. Das Erfassen von Craving am Versuchstier ist mit einem hohen experimentellen Aufwand verbunden und die verschiedenen Modelle werden derzeit noch kontrovers diskutiert [1]. Besonders geeignet scheinen jedoch Modelle zu sein, in denen Craving im Zusammenhang mit dem Rückfallverhalten untersucht wird. Alkohol trinkende Ratten, die mehrere Monate lang die freie Wahl zwischen Wasser und verschieden konzentrierten Alkohollösungen haben, zeigen nach einer längeren Abstinenzphase ein ausgeprägtes Rückfallverhalten, d.h., die Tiere trinken signifikant mehr Alkohol als zuvor [2,3] Dieses Phänomen, das als „Alkoholdeprivationseffekt" bezeichnet wird, lässt sich bei verschiedenen Tierspezies und beim Menschen beobachten. Der Alkoholdeprivationseffekt gilt heute als anerkanntes Modell für Rückfallverhalten und Craving nach Alkohol [4]. Ein vergleichbares Modell wurde auch für die intravenöse Selbstverabreichung von Heroin bei Ratten entwickelt. Bei ehemals heroinabhängigen Ratten führt bereits die Gabe einer sehr geringen Dosis von Heroin („priming“) oder eine kurze Stressepisode (Fußschock) zu einem stark ausgeprägten Rückfallverhalten [5]. Beide Modelle scheinen für die weitere 
Untersuchung der basalen Mechanismen von Craving und Rückfallverhalten gut geeignet.

Vorrangiges Ziel bei der Entwicklung einer Pharmakotherapie des Alkoholismus ist die Reduktion des Verlangens nach Alkohol zur Rückfallvermeidung. Seit einigen Jahren werden Acamprosat (Campral ${ }^{\circledR}$ ) und Naltrexon (Revia ${ }^{\mathrm{TM}}$ ) in den meisten europäischen Ländern in der Pharmakotherapie mit Erfolg klinisch eingesetzt [6]. Beide Substanzen unterdrücken auch in dem von uns entwickelten Modell dosisabhängig das Rückfallverhalten $[2,7,8]$. Hier erscheint es uns besonders bemerkenswert, dass nur die intermittierende Gabe von Naltrexon einen Rückfall verhindert, während die chronische kontinuierliche Gabe Craving sogar verstärkt und als Folge dann den Alkoholkonsum erhöht [8]. Diese Ergebnisse sprechen gegen den klinischen Einsatz von Naltrexon als Depotpräparat.

Anticravingsubstanzen entfalten ihre Wirkung über verschiedene Mechanismen. So blockiert Naltrexon Opiatrezeptoren auf Neuronen des mesolimbischen Belohnungssystems und hemmt dabei die akuten wie auch konditionierten Verstärkereigenschaften von Alkohol und Opiaten („positives Craving“) $[9,10]$, während Acamprosat vorwiegend mit dem glutamatergen N-Methyl-D-Aspartat-(NMDA-)System interagiert und zu deutlichen Veränderungen genomisch vermittelter Prozesse führt $[11,12]$. Acamprosat unterdrückt dabei sowohl akute als auch konditionierte Entzugssymptome („negatives Craving“) [13,14]. Es ist von großer Bedeutung für den therapeutischen Einsatz, dass diese Substanzen keine Substitutionsdrogen im herkömmlichen Sinne darstellen. Dies konnte für Naltrexon [16] und Acamprosat [15] in Drogendiskriminationsstudien nachgewiesen werden.

Obwohl die Pharmakotherapie im Alkoholismusbereich entscheidende Fortschritte gemacht hat, erscheint die Anwendung dieser Anticravingsubstanzen bei Opiatabhängigen wenig hilfreich. Der narkosegestützte Ultrakurzentzug mit Naltrexon und anschließender fortgesetzter Naltrexon-Medikation ist nach unseren Untersuchungen äußerst fragwürdig [17,18]. Mit der Verabreichung von Acamprosat hingegen konnten wir zeigen, dass die durch Morphin hervorgerufenen Sensitivierungsprozesse und Konditionierungsprozesse vollständig unterdrückt werden können. In dem bereits genannten HeroinRückfallmodell war Acamprosat jedoch wirkungslos [5].

Alternativ zur pharmakologischen Intervention bieten sich verschiedene Ansätze der Immunisierung an. Obwohl bei der Kokainimmunisierung bereits deutliche Fortschritte gemacht wurden [19] und erste klinische Studien begonnen haben, muss dieser Ansatz mit einiger Skepsis betrachtet werden. So wurde bei Rhesusaffen nach Immunisierung eine Gegenkompensation beobachtet - einige Tage nach der Immunisierung verabreichten sich die Affen mehr Heroin als davor.

\section{Literatur}

${ }^{1}$ Koob GF, Weiss F, Tiffany S, Zieglgänsberger W, Spanagel R. Animal models of craving. Alcohol Research \& Health 1999; 23: 233-236

2 Spanagel R, Hölter S, Allingham K, Landgraf R, Zieglgänsberger W. Acamprosate and alcohol: I. Effects on alcohol intake following alcohol deprivation in the rat. Eur J Pharmacol 1996; 305: 39-44
${ }^{3}$ Hölter SM, Engelmann M, Kirschke C, Liebsch G, Landgraf R, Spanagel R. Long-term ethanol self-administration with repeated withdrawal episodes changes ethanol drinking pattern and increases anxiety during withdrawal in rats. Behav Pharmacol 1998; 9: 41-48

${ }^{4}$ Spanagel R, Hölter SM. Long-term alcohol self-administration with repeated alcohol deprivation phases: An animal model of alcoholism? Alcohol and Alcoholism 1999; 34: 231-243

${ }^{5}$ Spanagel R, Sillaber I, Zieglgänsberger W, Corrigall WA, Stewart J, Shaham Y. Acamprosate suppresses the expression of morphine-induced sensitization in rats but does not affect heroin self-administration or relapse induced by heroin or stress. Psychopharmacology 1998; 139: 391-401

${ }^{6}$ Sass H, Soyka M, Mann K, Zieglgänsberger W. Relapse prevention by acamprosate: Results from a placebo controlled study in alcohol dependence. Arch Gen Psychiatry 1996; 53: 673-680

${ }^{7}$ Hölter SM, Landgraf R, Zieglgänsberger W, Spanagel R. Time course of acamprosate action on operant ethanol self-administration after ethanol deprivation. Alcohol Clin Exp Res 1997; 21: 862-868

${ }^{8}$ Hölter SM, Spanagel R. Effects of opiate antagonist treatment on the alcohol deprivation effect in long-term ethanol-experienced rats. Psychopharmacology 1999; 145: 360-369

${ }^{9}$ Spanagel R, Zieglgänsberger W. Anti-craving comounds: new pharmacological tools to study addictive processes. Trends Phamacol Sci 1997; 18: 54-59

${ }^{10}$ Spanagel R, Weiss F. The dopamine hypothesis of reward: past and current status. Trends Neurosci 1999; 22: 521-527

${ }^{11}$ Putzke J, Spanagel R, Tölle TR, Zieglgänsberger W. Acamprosate differentially alters PTZ and ethanol withdrawal-induced c-fos expression in the rat. Eur J Pharmacol 1996; 317: 39-48

${ }^{12}$ Rammes G, Mahal B, Putzke J, Parsons C, Spielmanns P, Pestel E, Spanagel R, Zieglgänsberger W, Schadrack J. The effects of the anti-craving/anti-relapse compounds acamprosate and memantine on the N-methyl-D-aspartate (NMDA) receptor in the rat brain. Neuropharmacology 2001; 40: 749-760

${ }^{13}$ Littelton JM. Acamprosate in alcohol dependence: how does it work? Addiction 1995; 90: 1197-1205

${ }^{14}$ Spanagel R, Putzke J, Stefferl A, Schöbitz B, Zieglgänsberger W. Acamprosate and alcohol: II. Effects on alcohol withdrawal in the rat 1996; 305: 45-50

${ }^{15}$ Spanagel R, Zieglgänsberger W, Hundt W. Acamprosate and alcohol: III. Effects on alcohol discrimination in the rat. Eur J Pharmacol 1996; 305: 51-56

${ }^{16}$ Spanagel R. The influence of opioid antagonists on the discriminative stimulus effects of ethanol. Pharmacol Biochem Behav 1996; 54: 645-649

${ }^{17}$ Spanagel R, Kirschke C, Tretter F, Holsboer F. Forced opiate withdrawal under anaesthesia prolongs and augments the occurence of withdrawal signs. Drug Alcohol Depend 1998; 52: 251-256

${ }^{18}$ Spanagel R. Is there a pharmacological basis for therapy with rapid opioid detoxification? The Lancet 1999; 354: 2017-2018

${ }^{19}$ Garrera MRA, Ashley JA, Wirsching P, Koob GF, Janda KD. A second - generation vaccine protects against the psychoactive effects of cocaine. Proc Natl Acad Sci USA 2001; 98: 1988-1992

\section{Rainer Spanagel}

Zentralinstitut für Seelische Gesundheit

Abteilung Psychopharmakologie

Postfach 122120

68072 Mannheim

E-mail: spanagel@as200.zi-mannheim.de 